\title{
Ampliaciones de una Mediateca Digital de Muestras de Laboratorio Histopatológico
}

\author{
Extensions of a Digital Library of Laboratory Samples Histopathology
}

\author{
"Rodolfo Esteban Ávila; "*Laura Alonso I. Alemany; ${ }^{* * * *}$ María Elena Samar; \\ ${ }^{* * * * *}$ Luisa Beatriz Buzzetti; ${ }^{* * * *}$ Gustavo Juri \& ${ }^{* * * *}$ Hugo Oscar Juri
}

\begin{abstract}
ÁVILA, R. E.; ALEMANY, L. A. I.; SAMAR, M. A.; BUZZETTI, L. B.; JURI, G. \& JURI, H. O. Ampliaciones de una mediateca digital de muetras de laboratorio histopatológico. Int. J. Morphol., 28(3):875-878, 2010.

RESUMEN: El objetivo general de acción de este proyecto es ampliar una mediateca digital consistente en imágenes de muestras histopatológicas obtenidas en la 2da. Cátedra de Biología Celular, Histología y Embriología de la Facultad de Ciencias Medicas Universidad Nacional de Córdoba, Argentina. Se proponen tres líneas de trabajo principales: 1- integración de imágenes y documentos científicos (abstract de artículos) mediante el uso de términos ontológicos, 2- articulación del listado de imágenes y documentos científicos con herramientas de acceso a la información multimedia (imágenes y texto), 3- incorporación de una traducción simi-automática ingles-español usando herramientas libres de traducción automática de lengua origen castellano a lengua de origen ingles. Creemos que la asociación de diferentes herramientas de gestión del conocimiento permitirán ampliar una mediateca digital de muestras de laboratorio correspondientes a resultados de nuestros de trabajos de investigación subsidiados por organismos oficiales. También para que los contenidos de este proyecto estén disponibles para un amplio usuario de las Ciencias de la Salud con diferentes niveles de alfabetización funcional. Por último nuestro proyecto permitirá la inclusión de profesionales de la Escuela de Bibliotecología y de la Facultad de Lenguas de nuestra Universidad Pública.
\end{abstract}

PALABRAS CLAVE: Mediateca digital; Laboratorio; Muestras; Histopatología.

\section{INTRODUCCIÓN}

Informática e Internet contribuyen a asociar por un lado el almacenamiento, gestión y suministro de grandes volúmenes de información y por el otro, la posibilidad de usar entornos de aprendizaje interactivos y autorregulados (Avila et al., 2009a, 2009b; 2009c).

Por otra parte, la indización o bases de datos en Internet permiten que se conozca la literatura científica y que pueda ser empleada por otros investigadores en cualquier lugar del mundo. Los index o bases de datos de mayor consulta, entre otros, son MEDLINE (sustituto del Index Medicus, de la NLM), Social Science Citation Index, Science Citation Index y Art Index (del ISI), Index Latinoamericano Periódica (de la UNAM, México) (Samar \& Ávila, 2005; Giglia \& Spinelli, 2010).
Actualmente, la automatización computacional ha incrementado la calidad del proceso documental y ha facilitado a los profesionales de las Ciencias de la Salud el acceso, vía Internet, a la búsqueda bibliográfica de "abstract" o resúmenes de trabajos científicos (Giglia \& Spinelli, 2009).

La histopatología es una disciplina que pertenece a las Ciencias Morfológicas donde la estructura de células, tejidos y órganos del cuerpo son examinadas con el microscopio. Las imágenes logradas en los laboratorio son procesadas digitalmente para su uso en la educación médica virtual usando internet (Avila \& Samar, 2004).

El objetivo general de acción de este proyecto es ampliar una mediateca digital consistente en imágenes de

* IIa. Cátedra de Biología Celular, Histología y Embriología. Facultad de Ciencias Médicas. Universidad Nacional de Córdoba. Córdoba, Argentina.

** Sección Ciencias de la Computación. Facultad de Matemática, Astronomía y Física Universidad Nacional de Córdoba. Córdoba, Argentina.

*** Cátedra A de Histología. Facultad de Odontología. II Cátedra de Biología Celular, Histología y Embriología. Facultad de Ciencias Médicas. Universidad Nacional de Córdoba, Córdoba, Argentina.

***** Cátedra de Informática Médica Facultad de Ciencias Médicas. Universidad Nacional de Córdoba. Córdoba, Argentina. 
muestras histopatológicas obtenidas en la 2da.Cátedra de Biología Celular, Histología y Embriología de la Facultad de Ciencias Medicas Universidad Nacional de Córdoba, Argentina.

\section{MATERIAL Y MÉTODO}

Nuestra mediateca digital posee imágenes de fotografías y diapositivas del archivo iconográfico y como resultados de proyectos de investigación científica subsidiados por la Secretaría de Ciencia y Tecnología de la Universidad Nacional de Córdoba y realizados en la 2da.Cátedra de Biología Celular, Histología y Embriología de la Facultad de Ciencias Medicas Universidad Nacional de Córdoba, Argentina.

Los archivos de imágenes tienen una extensión JPG y GIF. Se obtuvieron imágenes digitales de cortes histológicos de diferentes especies mediante un analizador de imágenes con el programa "Image Proplus", conectado a un fotomicroscopio Olympus Bx50 y a una cámara de video Sony.

Para el desarrollo del sitio Web se utilizarán las siguientes herramientas de diseño y programación: Microsoft Office SharePoint Designer: programa que permite tener una visión completa de un proyecto web. Este software tiene la facilidad de crear páginas web, e insertar dinamismo a las mismas a través de scripting y al mismo tiempo permite una administración global de todo el proyecto (hipervínculos, navegación, directorios, etc.). HTML: (HyperText Markup Language). Es un lenguaje para el formato de documentos de hipertexto que estructura un documento por medio de etiquetas (tags) que posteriormente interpretará el navegador (Mozilla Firefox, Netscape Navigator o Internet Explorer).

Integran nuestro equipo de trabajo: profesionales de las Ciencias de la Salud (Médicos, Odontólogos, Técnicos de Laboratorios), Informáticos, Bibliotecarios y Traductores.

\section{RESULTADOS}

Se proponen tres líneas de trabajo principales:

1- Integración de imágenes y documentos científicos (abstract de artículos) mediante el uso de términos ontológicos, Figura 1.
2- Articulación del listado de imágenes y documentos científicos con herramientas de acceso a la información multimedia usando internet (imágenes y texto), Figura 2.

En este sentido la ontología computacional nos permitirá cumplir nuestro trabajo mediante terminología específica usando el tesauro. El tesauro es una forma que tiene una base de datos para clasificar e indizar, empleando palabras clave, que en conjunto constituyen un vocabulario especializado de palabras jerárquicamente relacionadas entre sí.

3- Incorporación de una traducción semi-automática inglesespañol usando herramientas libres de traducción automática de lengua origen castellano a lengua de origen inglés, Figura 3.

\section{DISCUSIÓN}

Martínez de Sousa (mencionado por Roblero Carballo) dice que la Medioteca es "un lugar donde se tienen, organizan y ponen a disposición del público materiales audiovisuales y medios de comunicación social" "Se considera que es también la colección de registros, los espacios y recursos destinados a conservar y preservar para sus uso los documentos audiovisuales, lexivisuales y multimedia, así como los medios de comunicación social" (Roblero Carballo, 2002).

"Una de las características principales de la Mediateca es que no excluye ningún ambiente, por el contrario es incluyente en la medida en que acrecienta su capacidad tecnológica, considerando la conformación física y su correlación virtual". "De este modo, la Mediateca tiene una mayor virtud al producir conocimiento nuevo e interactuar en la construcción del mismo, pues lo hace de manera rápida y eficiente pues aplica oportunamente las tecnologías de información y comunicación además de que tiene la facultad de adaptarse a las necesidades del ser humano en cualquier ámbito en el que éste se desarrolle; estableciendo así una interfase propia entre éste y Mediateca"

Roblero Carballo expresa "Cada día crece más el valor del documento intangible y se hace necesario e indispensable debido a su interacción y su reciprocidad se habla de que son el vehículo de la memoria es por ello que la Mediateca desempeña una labor de suma importancia en todos los ámbitos y su importancia crece con la cantidad de información y conocimiento por ende los documentalistas, bibliotecarios, informáticos, ingenieros de la comunicación, etc. Están seriamente implicados ya 


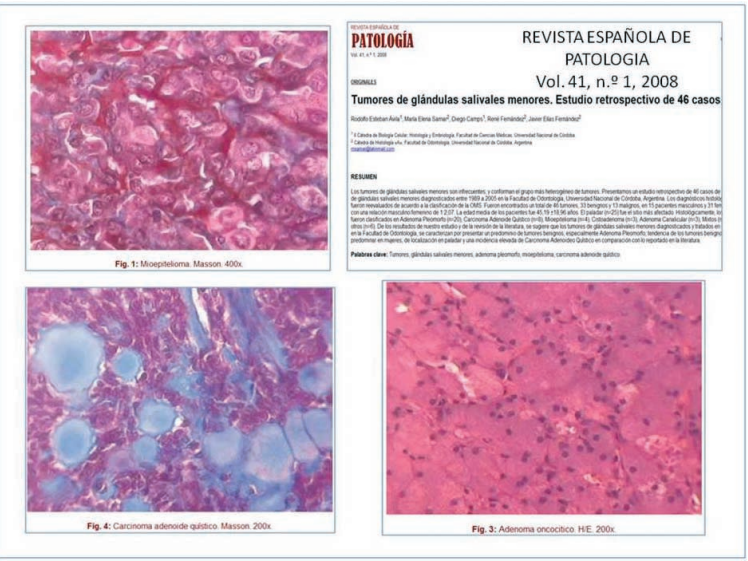

Fig. 1. Imagen que muestra un resumen e ilustraciones de la publicación en la Revista Española de Patología relacionadas con los tumores de glándulas salivares menores.

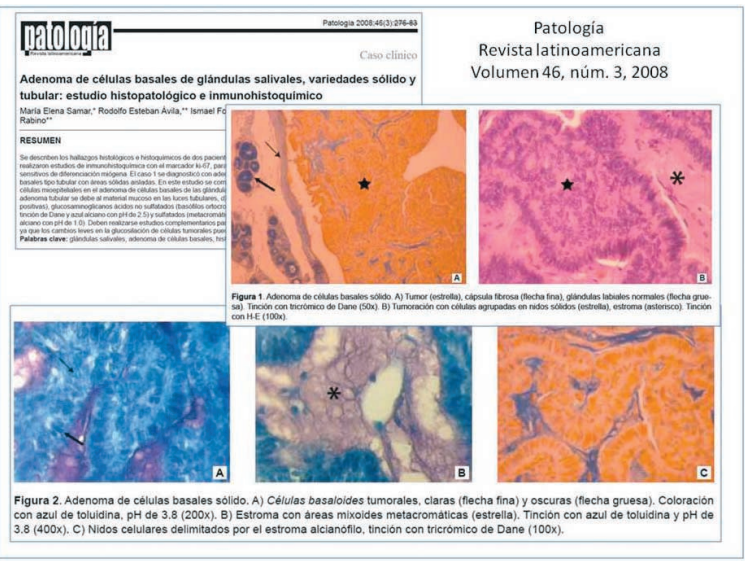

Fig. 2. Imagen que muestra un resumen e ilustraciones de la publicación en Patología Revista Latinoamericana relacionadas con adenoma de células basales de glándulas salivales.

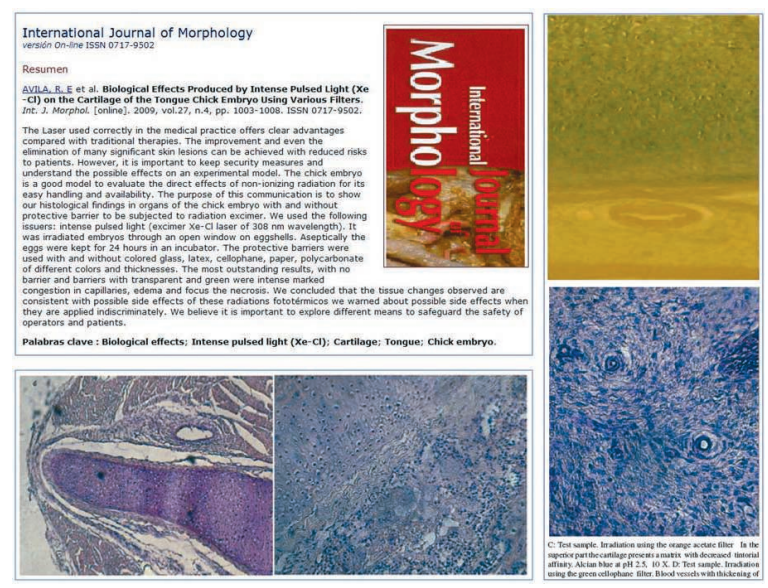

Fig. 3. Imagen que muestra un resumen e ilustraciones de la publicación en la Revista International Journal of Morphology relacionadas con los efectos biológicos producidos por la Luz Pulsada Intensa (Xe-Cl) sobre el cartílago lingual del embrión de pollo usando diversos filtros. que este medio abre igualmente innumerables perspectivas para el estudio y la investigación.

"Mediateca es una parte fundamental en los medios de información y documentación, debido a que en ella se conservan, reproducen y difunden materiales complementarios, tan solicitados en la nueva era de la información en la que nos desarrollamos, es por ello que la Mediateca debe ser un lugar adecuado y darle el mayor apoyo posible para su uso y difusión”.

Creemos que la asociación de diferentes herramientas de gestión del conocimiento permitirán ampliar una mediateca digital de muestras de laboratorio correspondientes a resultados de nuestros de trabajos de investigación subsidiados por organismos oficiales (Avila et al., 2008; 2009a, 2009b; 2009c; Samar et al., 2008).

También para que los contenidos de este proyecto estén disponibles para un amplio usuario de las Ciencias de la Salud con diferentes niveles de alfabetización funcional (Costello et al., 2003; Goldberg \& Dintzis, 2007; Silva Lópes \& Monteiro-Leal, 2003).

Por último nuestro proyecto permitirá la inclusión de profesionales de la Escuela de Bibliotecología y de la Facultad de Lenguas de nuestra Universidad Pública (Juri et al., 1991).

ÁVILA, R. E.; ALEMANY, L. A. I.; SAMAR, M. A.; BUZZETTI, L. B.; JURI, G. \& JURI, H. O. Extensions of a digital library of laboratory samples histopathology. Int. J. Morphol., 28(3):875-878, 2010.

SUMMARY: The overall objective of action of this project is to expand a digital media library consisting of images of histopathological samples obtained in the second department of Cell Biology, Histology and Embryology, Faculty of Medical Sciences, National University of Cordoba, Argentina. We suggests three lines of work: 1 - integration of images and scientific papers (abstract of articles) by using ontological terms, 2 - joint list of images and scientific papers with tools to access multimedia information (pictures and text ), 3 - incorporation of automatic translation English-Spanish using free software tools. We believe that the combination of different knowledge management tools allows expanding a digital media library of samples for laboratory results of our research work subsidized by government agencies. Also for the contents of this project to be available for any user of the Health Sciences with different levels of functional literacy. Finally our project will enable the inclusion of professionals from the School of Library and the Faculty of Languages of our public universities.

KEY WORDS: Digital library; Laboratory samples; Histopathology. 
ÁVILA, R. E.; ALEMANY, L. A. I.; SAMAR, M. A.; BUZZETTI, L. B.; JURI, G. \& JURI, H. O. Ampliaciones de una mediateca digital de muetras de laboratorio histopatológico. Int. J. Morphol., 28(3):875-878, 2010.

\section{REFERENCIAS BIBLIOGRÁFICAS}

Ávila, R. E.; Alonso, I.; Alemany, L. \& Samar, M. E. Herramientas de gestión del conocimiento en educación medica virtual histopatológica Patología. Patología, 47(4):374-5, 2009a.

Ávila, R. E.; Quiroga, M.; Ciucci, R.; Lucero, P.; Caballero, E.; Montes Tizca, A.; Mineo, J.; Maldonado, L. \& Samar, M. E. El laboratorio de investigación biomédica como "puente interactivo" entre el proceso de enseñanzal aprendizaje y la investigación de las neurociencias. Ini. Inv., 4:a2, 2009b. Disponible en: http://virtual.ujaen.es/ ininv/

Avila, R. E.; Samar, M. E.; Juri, H.; Femopase, G. A.; Hidalgo, M.; Ferrero, J. C.; Rinaldi, C.; Fonseca, I. \& Juri, G. Biological Effects Produced by Intense Pulsed Light $(\mathrm{Xe}-\mathrm{Cl})$ on the Cartilage of the Tongue Chick Embryo Using Various Filters. Int. J. Morphol., 27(4):1003-8, 2009c.

Avila, R. E. \& Samar, M. E. The Internet in the Medical Education: use of the virtual laboratory in the education of Morphologic Sciences. Technology and Health Care, 12(5):395, 2004.

Ávila, R. E.; Samar, M. E.; Camps, D.; Fernández, R. \& Fernández, J. E. Tumores de glándulas salivales menores. Estudio retrospectivo de 46 casos. Rev. Esp. Patol., 4:23-9, 2008.

Costello, S. S.; Johsnston, D. J.; Dervan, P. A. \& O`Shea, D. G. Development and evaluation of the virtual pathology slide: a new tool in telepathology. J. Med. Internet Res., 5(2):e11, 2003.

Giglia, E. \& Spinelli, O. PubMed in progress: further changes in the redesigned interface. Eur. J. Phys. Rehabil. Med., 46(1):113-5, 2010.

Giglia, E. \& Spinelli, O. PubMed reloaded: new interface, enhanced discovery. Eur. J. Phys. Rehabil. Med., 45(4):631-6, 2009.

Goldberg, H. R. \& Dintzis, R. The positive impact of teambased virtual microscopy on student learning in physiology and histology. Adv. Physiol. Educ., 31:2615, 2007.

Juri, H.; Sipowicz, O.; Avila, R. E.; Hernández, D. \& Palma, A. Propuesta para la enseñanza y aplicación de la informática en la Escuela de Medicina. Rev. Fac. Cienc. Méd. Córdoba, 49(1): 39-42, 1991.

Roblero Carballo, M. A. EL PROYECTO DE LA MEDIATECA DEL IPN, 2002. Disponible en: http:// azul.bnct.ipn.mx/iv_aniv/panel5_2.htm

Samar, M. E. \& Ávila, R. E. La búsqueda bibliográfica y la recuperación de la información en las ciencias. Archivos de Educación Médica, 1(1):6, 2005.

Samar, M. E.; Ávila, R. E.; Fonseca, I.; Ferraris, R. V. \& Rabino, M. Adenoma de células basales de glándulas salivales, variedades sólido y tubular: estudio histopatológico e inmunohistoquímico. Patol., 46(3):276-83, 2008.

Silva Lópes, V. W. \& Monteiro-Leal, L. Creating a HistologyEmbryology free digital image database using high-end microscopy and computer techniques for on line biomedical education. Anat. Rec., 273:126-31, 2003.

Dirección para correspondencia:

Prof. Dr. Rodolfo E. Avila.

Calle Catamarca 1546 (500)

Córdoba

ARGENTINA

Email: ravila@cmefcm.uncor.edu

Recibido : 25-04-2010

Aceptado: 28-06-2010 